IdeAs

Idées d'Amériques

8 | 2016

Ressources minières dans les Amériques: Mutations d'un continent

Justin Vaïsse, Zbigniew Brzezinski. Stratège de l'empire Paris, Odile Jacob, 2015, 422 pages

Isabelle Vagnoux

(2) OpenEdition

Journals

Édition électronique

URL : https://journals.openedition.org/ideas/1825

DOI : $10.4000 /$ ideas. 1825

ISSN : 1950-5701

Éditeur

Institut des Amériques

Référence électronique

Isabelle Vagnoux, « Justin Vaïsse, Zbigniew Brzezinski. Stratège de l'empire », IdeAs [En ligne], 8 | 2016,

mis en ligne le 06 janvier 2017, consulté le 18 octobre 2022. URL : http://journals.openedition.org/

ideas/1825; DOI : https://doi.org/10.4000/ideas.1825

Ce document a été généré automatiquement le 18 octobre 2022.

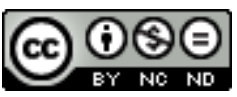

Creative Commons - Attribution - Pas d'Utilisation Commerciale - Pas de Modification 4.0 International - CC BY-NC-ND 4.0

https://creativecommons.org/licenses/by-nc-nd/4.0/ 


\title{
Justin Vaïsse, Zbigniew Brzezinski. Stratège de l'empire
}

Paris, Odile Jacob, 2015, 422 pages

\author{
Isabelle Vagnoux
}

\section{RÉFÉRENCE}

Justin Vaïsse, Zbigniew Brzezinski. Stratège de l'empire, Paris, Odile Jacob, 2015, 422 pages

Spécialiste de relations internationales et de politique étrangère américaine, l'historien Justin Vaïsse signe là un remarquable ouvrage, passionnant de bout en bout. Au fil d'un texte dense, parfaitement documenté, largement rédigé à partir d'archives ou d'entretiens avec Zbigniew Brzezinski, le lecteur se familiarise avec cet éminent "stratège ", au cœur de la politique étrangère américaine depuis près de soixante ans.

1 L'histoire débute avec l'enfance du jeune Zbigniew, fils de diplomate polonais en poste au Canada, qui suit attentivement, par la presse et la radio, l'invasion de son pays par les troupes de Hitler en 1939 puis le déroulement de la Seconde Guerre mondiale. Ainsi naît sa passion pour les affaires internationales qui dominera toute sa vie, de ses années d'universitaire à Harvard puis à Columbia, en tant que soviétologue, à l'éminent expert, en passant par la Maison Blanche, à la tête du Conseil national de sécurité (NSC) de Jimmy Carter. Mais l'on perd très vite de vue l'homme privé qui s'efface derrière l'expert et le stratège politique, dans une étude à tiroirs qui, entre autres, plonge le lecteur dans « l'université de guerre froide » des années 1950 qui peu à peu remplace le monde des affaires comme conseil des présidents, dans la relation avec l'autre grand intellectuel et stratège des relations internationales, Henry Kissinger, ou encore dans le fonctionnement de l'Administration Carter et du président lui-même.

2 À la fin des années 1950, les grands discours musclés mais non suivis d'effet des Républicains à l'égard des Soviétiques le déçoivent et font naître en lui un "faucon ", 
notamment envers Cuba et le Vietnam. Endiguer Moscou, «expansionniste opportuniste ", devient son mot d'ordre. L'auteur résume l'évolution tactique à l'égard de l'URSS en quatre phases : la déception face aux promesses républicaines non tenues $\mathrm{du}$ roll back; l'exploitation de l'attraction naturelle des pays d'Europe de l'Est pour l'Occident, sorte de détente avant l'heure; le renforcement des alliances; le retour à l'endiguement, qui s'accentue à partir de 1978. Mais trois piliers demeurent constants : la nécessité de maintenir des moyens militaires puissants ; le rôle des nationalismes et des divisions du bloc soviétique; le soutien idéologique, notamment par le biais des radios.

3 Brzezinski se tourne vers les Démocrates et s'implique personnellement dans la campagne de Kennedy, mais la collaboration avec la Maison Blanche demeure réduite. Elle se resserre sous Johnson, avec un poste au sein du département d'État, au Policy Planning, mais le costume est trop étroit pour lui. Il contourne alors les voies hiérarchiques pour donner son avis directement au président. Justin Vaïsse souligne son influence, notamment sur le discours «Est-Ouest » du président, en octobre 1966. En 1968, Brzezinski conseille le candidat démocrate Humphrey sur les questions internationales. La victoire de Nixon l'éloigne du pouvoir mais, infatigable, il transmet notes et idées au nouveau président. Avec Kissinger à la tête du NSC, une page symbolique se tourne. L'establishment cède le pas devant « l'élite professionnelle, dont Kissinger et Brzezinski sont les précurseurs ». J. Vaïsse bat en brèche toute idée de rivalité entre les deux hommes et s'appuie sur les archives pour peindre une relation cordiale, riche de nombreux échanges épistolaires, où la concurrence n'est pas absente, «mais n'apparaît jamais dominante ». Tous deux se caractérisent d'ailleurs par leur absence de sectarisme, dans un environnement de plus en plus politiquement polarisé. Lorsque Kissinger arrive au pouvoir, la relation se complexifie; si le dialogue reste ouvert, Brzezinski ne ménage pas ses critiques en public. La tension est palpable lors des campagnes de 1972 et 1976, année où les échanges prennent fin. Le parcours des deux hommes apparaît remarquablement similaire, toutefois Kissinger « a pris grand soin de cultiver sa légende", publiant 4000 pages de mémoires contre 573 pour Brzezinski qui, manquant de cette séduction devenue la marque de Kissinger, subit « un traitement médiatique brutal ", suscitant presque toujours des "réactions tranchées d'aversion ou d'admiration ». Là où Kissinger préfère la stabilité et ne s'intéresse qu'aux affaires diplomatiques et militaires, dans une "vision sélective du monde", Brzezinski accueille le changement historique, qu'il voit comme une force positive pour l'Amérique, et s'intéresse aux tensions sociopolitiques, aux bouleversements technologiques, aux idéologies.

4 En fondant, en 1973, avec David Rockefeller, la Commission trilatérale, organisation privée regroupant les élites américaines, européennes et japonaises, Brzezinski s’attelle à concrétiser une de ses grandes idées : dépasser les considérations intérieures afin de forger une communauté des nations développées au sein d'un ordre de plus en plus globalisé, à l'ère de la « révolution technétronique ». Car, l'Amérique ayant « besoin de partenaires égaux », il croit fermement à l'alliance avec une Europe forte et unie et un Japon intégré dans le monde des grandes puissances. C'est là qu'il rencontre le gouverneur Carter, dont il devient le principal conseiller en politique étrangère, soulignant la "relation symbiotique » entre sa campagne et la Commission, dans la vision des alliances, d'une meilleure stabilité des relations Nord-Sud, de la recherche d'une détente différente avec l'URSS, plus réciproque et faisant intervenir la «carte chinoise ». Selon J. Vaïsse, Carter est façonné par les experts de la Trilatérale, très 
nombreux dans son gouvernement aux postes les plus élevés. Nommé à la tête du NSC en 1977, et déçu par l'absence d'action de la Trilatérale qui rencontre peu d'écho en Europe et au Japon, Brzezinski sort peu à peu du cadre États-Unis/Europe/Japon pour prendre en compte d'autres pays à l'influence croissante. La Commission prend fin en 1978, cédant le pas à d'autres structures internationales.

5 Au fil des quatre années passées à la Maison Blanche, le ciment qui unissait Brzezinski et Carter s'amenuise sous le poids du pouvoir et des luttes d'influence intestines, notamment entre le département d'État, que dirige Cyrus Vance, et le NSC, rivalité que $\mathrm{J}$. Vaïsse nuance toutefois. Le désaccord entre les deux hommes ne survient que graduellement et pas sur tous les dossiers. La coopération entre eux reste bonne sur le canal de Panama, le Proche Orient, la gestion des crises en Afrique australe. L'affrontement est en revanche indéniable sur l'orientation générale de la politique étrangère américaine, car Vance souhaite avant tout poursuivre la détente, alors que Brzezinski cherche à contrer l'URSS. Le problème, c'est bien le président lui-même, son indécision, son incapacité à trancher entre ces deux approches. Brzezinski jouit cependant d'un net avantage : son omniprésence auprès de Carter et le contrôle qu'il exerce sur le processus de rédaction des discours présidentiels en matière de relations internationales. Le durcissement des positions de Carter, en fin de mandat, marque sa victoire idéologique.

Le bilan que dresse Justin Vaïsse de "l'Administration Brzezinski»/Carter est, à nouveau, nuancé, avec des demi-succès : le traité SALT 2, abouti trop tardivement, ne pourra se concrétiser; la politique des droits de l'homme, aux résultats mitigés. Les échecs indiscutables: l'Iran et la prise d'otages américains (Brzezinski prônait un soutien à un coup d'État militaire mais ne fut pas entendu), le coup de froid avec l'URSS (J.Vaïsse réfute cependant l'accusation selon laquelle Brzezinski serait un " déclencheur de guerre froide »), l'Afghanistan. Mais aussi des succès historiques : les traités sur le canal de Panama, les accords de Camp David scellant la paix entre Israël et l'Egypte, la normalisation des relations diplomatiques avec la Chine. En bref, autant de " décisions courageuses et coûteuses électoralement " pour J. Carter qui faciliteront le travail de R. Reagan. Justin Vaïsse réhabilite ainsi une présidence injustement passée à la postérité comme l'une des pires présidences américaines de la deuxième partie du XXe siècle.

7 Qu'en est-il des «prophéties » de Brzezinski ? Pour l'auteur, il s'est parfois trompé (le bilan de ses prévisions sur le Vietnam est mitigé, il ne perçoit pas le potentiel réformateur de Gorbachev), mais il a aussi souvent visé juste (Afrique, Japon), même si ses intuitions ont pu se concrétiser en décalage (par exemple, l'avènement des technologies de l'information ou l'ère technétronique). Il n'a jamais adhéré à la thèse du déclin de l'Occident, convaincu que le système soviétique était voué à disparaître, mais aussi que le nationalisme grand-russe assurerait sa persistance. Il voit juste sur les réformes économiques menées par la Chine mais se trompe sur l'ouverture politique qu'elles seraient susceptibles de générer. Au total, un «bilan en demi teinte mais plutôt positif».

8 Brzezinski s'est imposé comme le «stratège par excellence de l'empire américain ». Depuis 1961, il aura conseillé personnellement tous les présidents, sauf George W. Bush, dont il critique l'absence de vision stratégique élaborée et le penchant néoconservateur. Depuis son départ de la Maison Blanche, en 1981, Brzezinski continue de peser sur les débats importants de Washington. Une formidable longévité pour celui 
qui n'a exercé de responsabilités de haut niveau que pendant quatre années. J. Vaïsse attribue cette influence exceptionnelle à sa production intellectuelle, à l'attention qu'il prête aux "forces profondes", à son indépendance d'esprit. Brzezinski n'est pas l'homme d'un parti, il apparaît « finalement inclassable, farouchement indépendant et irréductible à une école de pensée particulière ».

\section{AUTEURS}

\section{ISABELLE VAGNOUX}

Isabelle Vagnoux est professeur des Universités à Aix Marseille Université (LERMA).

isabelle.vagnoux@univ-amu.fr 